\title{
Notiz über die Antennendrüse der Cladoceren.
}

Von

\author{
F. E. Rühe (Berlin).
}

Mit 2 Abbildungen im Text.

Im vorletzten Heft dieser Zeitschrift ${ }^{1}$ ) findet sich ein Aufsatz von Dr. Langhans über "Eine rudimentäre Antennendrüse bei Cladoceren als Ergebnis der Vitalfärbungsmethode", in dem Langhans zu dem Ergebnis gelangt, daß die zwei von Fischel bei Cladoceren mittels der Vitalfärbungsmethode dargestellten und als "Drüsen unbekannter Natur" beschriebenen Organe als Endsäckchen der Schalendrüse bzw. Rest der Antennendrüse anzusprechen sind. $\mathrm{Zu}$ diesem Nachweis möchte ich die historische Bemerkung machen, daß Kowalevsky gemeinsam mit Metschnikoff schon im Jahre 1889 das Vorhandensein dieser beiden Drüsen festgestellt hat. Er spricht sich darüber folgendermaßen aus ${ }^{2}$ ): Herr E. Metschnikoff fütterte verschiedene Phyllopoden, namentlich Daphniden, Branchipus und Artemia mit Karmin und fand, daß bei allen diesen Crustaceen das Endsäckchen ihrer Schalendrüse und ein kleiner Rest der Antennendrüse sich mit Karmin färben. Ich habe diese Versuche wiederholt und kann dieselben vollständig bestätigen und so weit vervollständigen, daß ich alle diese Crustaceen mit Lakmus fütterte und eine rote Färbung des Endsäckchens erzielte." Diese kurze Notiz, die leider nicht angibt, bei welchen Daphniden sich ein Rest der Antennendrüse färbte, konnte leicht übersehen werden. Dann aber. teilte L. Bruntz in einer größeren Arbeit ${ }^{3}$ ) im Jahre 1903 ähnliche Versuche mit und kam zu sehr interessanten Ergebnissen über Maxillen- und Antennendrüse der Cladoceren, auf die ich besonders auf-

1) Internat. Revue d. ges. Hydrobiol. u. Hydrogr, Bd. Il (Heft. 1 1. 2), S. 182-185, Taf. 12.

2) Kowalevsky, A., Beitrag zur Kenntnis der Exkretionsorgane. Biol. Centralbl. 1889, Bd. IX, S. 33.

3) Contribution à l'étude de l'excrétion chez les Arthropodes, (Thèses de la Faculté des Sciences Univers. Nancy.) 1903, p. 234--236. 
merksam machen möchte. Ich zitiere, da die betreffende Arbeit schwer zugänglich sein dürfte, die zum Vergleich mit den Ergebnissen von Fischel und Langhans wichtigsten Sätze. "Grâce à la transparence des téguments, on peut reconnaître la place exacte de ces organes (Antennendrüse) disposés symmétriquement à chaque côté du tube digestif à l'endroit où sa courbure est la plus prononcée, immédiatement au-dessous des coecums. Ils sont situés à la base et dans le prolongement des antennes. Des coupes sériées montrent qu'ils sont constitués chacun par un amas de trois ou quatre cellules éliminant le carminate. Ces cellules sont grosses, régulières, ne possèdent qu'un seul noyáu, et sont en tous points semblables à celles de l'épithélium du saccule du rein maxillaire. Mes préparations me conduisent à considérer ces formations comme des organes excréteurs clos, représentant par conséquent un rein d'accumulation ou de transformation. En effet, je n'ai jamais vu, sur des coupes, un de ces organes déboucher, soit directement au dehors, soit dans un canal excréteur constituant un labyrinthe. D'un autre côté, si on place dans de l'eau pure des Daphnies dont les organes excréteurs sont bourrés de carminate éliminé, on retrouve encore du carminate dans le rein antennaire longtemps après la décoloration $\mathrm{du}$ saccule du rein maxillaire. Considérant la position spéciale de ces groupes de cellules excrétrices, je crois qu'ils correspondent à des saccules de reins antennaires dont les labyrinthes seuls se seraient atrophiés pendant le développement postlarvaire."

Diese Angaben von Bruntz stehen im schönsten Einklang mit denen von Fisehel und Langhans, ich weise nur hin auf die von den beiden letzteren betonte "Unveränderlichkeit der Farbreaktion" der Antennendrüse. Die von Langhans offen gelassene Frage betreffend das Vorhandensein eines Ausführganges der Antennendrüse wird von Bruntz schon dahin entschieden, dal ein solcher nicht vorhanden ist. Bruntz gibt dann noch eingehende Angaben über die Histologie von Endsäckchen und Labyrinth der Schalendrüse, die er nach denselben Methoden wie die Antennendrüse gefärbt und geschnitten hat. Aufmerksam machen möchte ich noch auf die nach gleicher Methode gewonnenen Resultate an Artemia salina, an der Bruntz außer der Schalen- und Antennendrüse noch sog. Nephrophagocyten studiert hat.

Leider hat Bruntz aber seiner Arbeit keine Figuren, die die Lage der Antennendrüse veranschaulichen könnten, beigegeben, was um so mehr vermißt wird, als sein Ausdruck: "au dessous des coecums", ebenso wie der von Langhans: "unter oder in der Basis der Ruderantenne" an Deutlichkeit zu wünschen übrig läßt, da beide nicht angeben, wie sie den Körper ihrer Untersuchungsobjekte orientiert wissen wollen. Da auch aus Langhans' Photographie die Lage der Antennendrüse nicht genau zu ersehen ist und in Fischels Figuren der zur Lagebestimmung wichtige 
Fornixrand nicht eingezeichnet ist, habe ich selbst versucht über die Lage der Antennendrüse ins Klare zu kommen und will im folgenden über mein Ergebnis kurz berichten.

Als Objekte dienten mir Daphnia pulex und Simocephalus vetulus, und ich färbte die Exkretionsorgane, indem ich die Tiere in schwachen Lösungen von Neutralrot, Methylenblau, Karmin und karminsaurem Ammoniak leben ließ. Bei Daphnia pulex gelang es mir trotz längerer Versuche mit keinem dieser Farbstoffe eine Antennendrüse zu finden, was um so bemerkenswerter ist, als Fischel und Langhans dieselbe bei Daphnia magna fanden. Auch bei entwickelteren Embryonen, die ich aus dem Brutraum herauspräparierte und die bei Simocephalus die Antennendrüse recht deutlich zeigten, fand ich dieselbe nicht. ${ }^{1}$ )

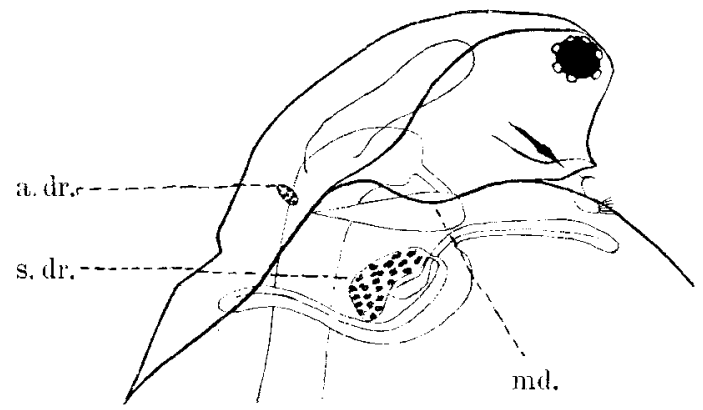

Fig. 1. Simocephalus vetulus. Seitenansicht.

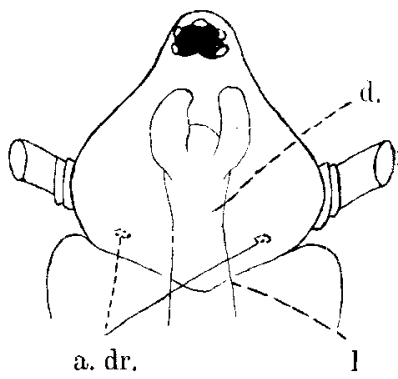

Fig. 2. Dorsalansicht.

Dagegen gelang es mir, dieselbe unzweifelhaft bei Simocephalus vetulus; festzustellen, wo sie auch schon Bruntz gefunden hat. Bei Färbung mit Neutralrot kann man allerdings ohne vorherige Kenntnis der Lage und Form der Schalendrüse bei den vielen Bindegewebs- und Fettkörperzellen, die sich intensiv färben, im Zweifel sein, ob und welche Zellgruppe man: als Antennendrüse anzusprechen hat. Doch fällt auch hier schon die Antennendrüse bei der Ahnlichkeit ihres Farbtones mit dem des Endsäckchens der Schalendrüse in die Augen. Bei Färbung mit Methylenblau und karminsaurem Ammoniak indessen ist ihre Lage ganz eindeutig festzustellen. Das karminsaure Ammoniak namentlich (das übrigens ebenso wie Karmin, mit dem ich keine Erfolge hatte, kein Vitalfarbstoff im sinne Fischels ist) eignet sich vorzüglich zur Darstellung des Endsäckchens der Schalendrüse und der Antennendrüse, da sich mit ihm nur diese beiden

1) Fischel (Lntersuchungen über vitale Fårbung an Süßwassertieren, Internation. Revue der ges. Hydrobiol. 1. Hydrogr, Bd. 1, H. 1-2, S. 89) behauptet übrigens, daB die Antennendrüse bei jungen Tieren von Daphnia magna gewöhnlich fehlt. Das trifft für Simocephalus vetulus sicher nicht zu. 
Exkretionsorgane und der Darm rot färben. Das Ergebnis dieser Färbung ist folgendes. Die Antennendrüse von Simocephalus vetulus ist ein länglichovales Gebilde, das nach der Zahl der gefärbten Granula zu schließcn aus 7 bis 10 Zellen besteht (Bruntz hat auf Schnitten nur 3 bis 4 Zellen gefunden) und eine geschlossene Drüse darstellt. Sie liegt dicht unter der Cuticula dorsal von der Anheftungsstelle der Mandibel, also nicht mehr in der Schalenduplikatur der Brust, sondern in der des Kopfes ${ }^{1}$ ). (Die Lage kann übrigens - allerdings nur scheinbar - variieren, was auf versehiedene Lagen des Tieres zurückzuführen ist.) Diese Lage der Antennendrüse ist deshalb zu betonen, weil auch bei BranchiopodenLarven die Antennendrüsen in dem Kopfteile vor der Ursprungsstelle der Brustschalenduplikatur liegt. Aus den bisher gegebenen Figuren und Beschreibungen mußte man den Eindruck gewinnen, daß dieselbe in der Brustschale läge, was eine Homologisierung mit der Antennendrüse der Branchiopoden ausschlöße. Damit glaube ich, die Lage der Antennendrüse für Simocephalus vetulus festgelegt zu haben. Es bleibt vorläufig eine offene Frage, ob die Antennendrüse anderer Cladoceren ebenfalls in der Kopfschale liegt. Hiermit ist das Vorhandensein einer Antennendrüse für zwei Cladocerenspecies festgestellt: Daphnia magna und Simocephalus vetulus. Dieselbe scheint zu fehlen bei: Daphnia pulex und (nach Langhans) Sida, Leptodora, Daphnia longispina.

\section{Figurenerklärung.}

a. dr. Antennendrüse. s. dr. Endsäckchen der Schalendrüse. md. Mandibel. 1. Trennungslinie zwischen Kopf- und Brustschale. d. Darm.

1) Langhans äußert sich nicht darüber, ob die Antennendrüse in der Kopf- oder Brustschale liegt. 